\title{
A stereo illusion induced by binocularly presented gratings: Effects of number of eyes stimulated, spatial frequency, orientation, field size, and viewing distance
}

\author{
J. VERNON ODOM and GUNG-MEI CHAO \\ West Virginia University Medical Center, Morgantown, West Virginia
}

\begin{abstract}
We report that the binocular presentation of gratings under appropriate conditions results in an illusion of depth. Multiple levels of depth are seen simultaneously, distinguishing the illusion from the wallpaper illusion. The levels are not overlapping but are $180^{\circ}$ out of phase relative to one another. The conditions under which the illusion is visible and under which it disappears are presented. Probable causal mechanisms, the correspondence problem, the horopter, and differential image projections of large tangential stimuli are discussed.
\end{abstract}

Recently, while examining binocularly a large photographic print of square-wave gratings, we observed a sensation of several nonoverlapping, discrete levels of depth. The usual appearance of the illusion, if one's eyes fixated at eye level or slightly above, was that of a distant ellipse surrounded by more proximal ellipses. Figure 1 provides an illustration of this percept. With more eccentric fixation, above or below, to left or right, one saw a succession of planes that receded into the veridical plane at their distal ends. The number of planes tended to increase with more eccentric fixation.

The illusion we observed was quite different from those obtained by using periodic stimuli (reported by, e.g., Brewster, 1844/1983, and Blakemore, 1970) in that we observed several levels of depth simultaneously, with the levels being displaced in the visual field relative to one another rather than being a single plane that changed its apparent depth or was tilted. It differed from the illusion observed by Boothroyd and Blake (1984) in that the gratings were presented binocularly and different portions of the figure were at different depths. Thus, the illusion is most similar to the one described by Piggins (1978), which also had several simultaneously present, nonoverlapping levels of depth. However, he employed dichoptically presented gratings of different orientations, whereas we employed a single, binocularly viewed grating.

This research was partially supported by grants from NEI (EY04806) and the Knights' Templar Eye Foundation, Inc., and by an unrestricted departmental grant from Research to Prevent Blindness, Inc., New York, NY. The authors wish to acknowledge the friendly skepticism and helpful comments of Stuart Anstis, Patricia Apkarian, Michael Fendick, Marc Green, John Siegfried, Henk Spekreijse, and Christopher Tyler. Portions of the data presented here were presented at the annual meetings of the Optical Society of America, Washington, DC, October 1985, and the Society for Neuroscience, Dallas, TX, November 1985, and appeared in abstract form in the Joumal of the Optical Society of America A, 1985, 2,45 , and the Society for Neuroscience Abstracts, 1985, 11, 652. The authors' mailing address is: Department of Ophthalmology, West Virginia University Medical Center, Morgantown, WV 26506.
Below we present the results of three experiments that examined these depth sensations as a function of monocular or binocular viewing, fundamental spatial frequency of the grating, orientation, field size, and viewing distance. The results indicate that the illusion depends on retinal disparity and probably results from an inappropriate solution by the visual system to the correspondence problem (Julesz, 1971; Koffka, 1935).

\section{EXPERIMENT 1}

\section{Method}

Three subjects participated in the experiment, J.V.O., G.M.C., and R.R.H. G.M.C. and R.R.H. were emmetropic, and J.V.O. was corrected to $6 / 6(20 / 20)$. All 3 subjects had normal stereothresholds and had no deviations of the alignment of the two eyes. Interpupillary distances for J.V.O., R.R.H., and G.M.C. were $6.6,6.3$, and $6.1 \mathrm{~cm}$, respectively.

The stimuli were eight photographic prints of square-wave gratings that, at a viewing distance of $35.6 \mathrm{~cm}$ (14 in.), had fundamental spatial frequencies of $0.3,0.6,0.9,1.1,1.5,3.2,4.2$, and $5.7 \mathrm{cy}-$ cles/degree (cpd) and a field size that subtended $43^{\circ} \times 53^{\circ}$ $(11 \times 14$ in.). The mean luminance of the 0.28 -cpd grating was $150 \mathrm{~cd} / \mathrm{m}^{2}$; its contrast was $75 \%$. The highest frequency grating was also presented at a distance of 53 and $86 \mathrm{~cm}$ (21 and $34 \mathrm{in}$.). These two stimulus conditions resulted in gratings of 8.2 and $13.3 \mathrm{cpd}$ with field sizes of $29^{\circ} \times 37^{\circ}$ and $18^{\circ} \times 23^{\circ}$, respectively.

The 10 spatial frequencies were presented at seven orientations: $0^{\circ}, 15^{\circ}, 30^{\circ}, 45^{\circ}, 60^{\circ}, 75^{\circ}$, and $90^{\circ}$. The orientation values refer to the deviation from the vertical. Thus, $0^{\circ}$ refers to the vertical, $90^{\circ}$ to the horizontal, and $45^{\circ}$ to an orientation midway between the vertical and the horizontal. All deviations from the vertical tilted to the right. The center of the stimulus did not change as orientation was varied.

The subjects viewed all stimuli at all seven orientations as they fixated the stimulus either with both eyes open or with the left eye closed. Although the subjects were not instructed to fixate any particular locus on the stimulus, they reported a tendency to fix below the center of the display because it improved the clarity of the illusion. The order of experimental conditions was counterbalanced across subjects and sessions. For each experimental condition, each subject made five ratings. The subjects were instructed to view the 


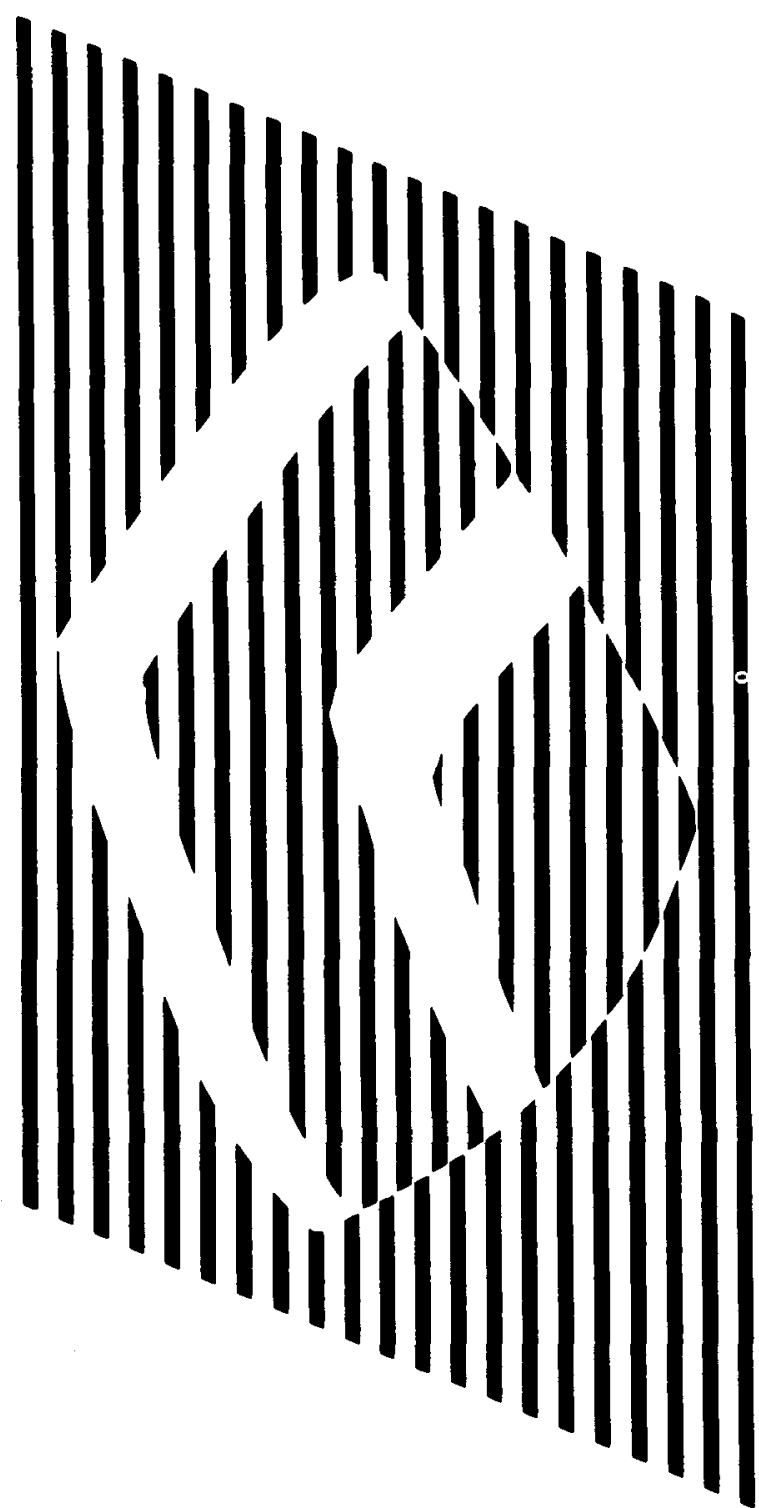

Figure 1. A representation of the illusion. Binocular fixation of a grating at or slightly above eye level and the grating's center results in a sensation of a central region that is at approximately the depth of the stimulus surrounded by other more peripheral regions, each of which appears closer to the subject. The exact sensation varies, depending on locus of fixation, spatial frequency, and field size.

print and state first if they saw more than one level of depth in the print. If they saw more than one level of depth, they were to report the number of levels $(N \geq 2)$. The criterion for considering a percept as having more than one level of depth was discussed with the subjects. The subjects were to indicate perceiving multiple levels of depth only if an edge or discontinuity separated the distinct levels of depth. A percept was considered to be a single plane if it had no sharp discontinuities in depth even if it "shimmered," appeared wavy, or undulated so that some parts appeared closer than others, or if the individual bars appeared tilted in depth or at different levels.
An indefinite sensation of depth, as though two levels were simultaneously present or one seen through the other, but with no edge separating one level from the other, was not classified as seeing more than one level. The 3 subjects made five ratings of perceived depth in each of 140 experimental conditions ( 2 viewing conditions $\times 10$ spatial frequencies $\times 7$ orientations).

\section{Results}

Under monocular viewing conditions, none of the 3 subjects reported seeing more than one level of depth. The sensation of a single depth plane with monocular viewing was unaltered by spatial frequency or orientation. Under monocular viewing, sensations of shimmer, movement, waviness, and undulation were reduced but persisted.

Under binocular viewing conditions, multiple levels of depth were seen by all 3 observers under some conditions. The percentage of trials on which individual subjects were able to see two or more levels of depth is presented as a function of spatial frequency and orientation in Figure 2. Despite some individual variation, the pattern of results is similar across subjects. In Figure 3, the responses of the 3 subjects are averaged to demonstrate the effects of spatial frequency on the percentage of trials on which two or more levels of depth were seen. One sees several levels of depth on $50 \%$ or more of the trials between about 1 and 9 cpd. Multiple levels of depth were seen most frequently at about $4 \mathrm{cpd}$. The effects of orientation are presented in Figure 4 for the spatial frequencies that resulted in subjects' seeing two levels of depth on $70 \%$ or more of the trials at one or more orientations. The data are fitted using the logistic function because the relationship between orientation and percentage of trials on which two levels were seen is nonnormal (Berkson, 1951; Reed \& Berkson, 1929). The orientation at which subjects reported seeing two or more levels of depth on $50 \%$ or more of the trials varied with spatial frequency. For 1.5-cpd gratings, two or more levels of depth were seen $50 \%$ or more of the time if the orientation was $45^{\circ}$ or less from the vertical. At $3.2,4.2$, and $5.7 \mathrm{cpd}$, the $50 \%$ point was about $60^{\circ}$ from the vertical. More than one level of depth was never perceived with binocular viewing of gratings if the gratings were oriented horizontally $\left(90^{\circ}\right)$.

The number of levels seen also varied as a function of spatial frequency and orientation. The mean number of levels reported averaged across subjects in the binocular viewing conditions is presented in Figure 5. In general, the largest mean number of levels of depth was seen at 4.2 and $3.2 \mathrm{cpd}$. At 1.5 and $5.7 \mathrm{cpd}$, two or more levels were consistently seen. Likewise, as grating orientation deviated from the vertical, fewer levels of depth were seen.

To generate higher spatial frequencies (8.2 and $13.3 \mathrm{cpd}$ ), we increased the viewing distance from the stimulus. Naturally, this also reduced the field size of the stimulus. Therefore, we conducted Experiments 2 and 3 to determine the likelihood that increased viewing distance 


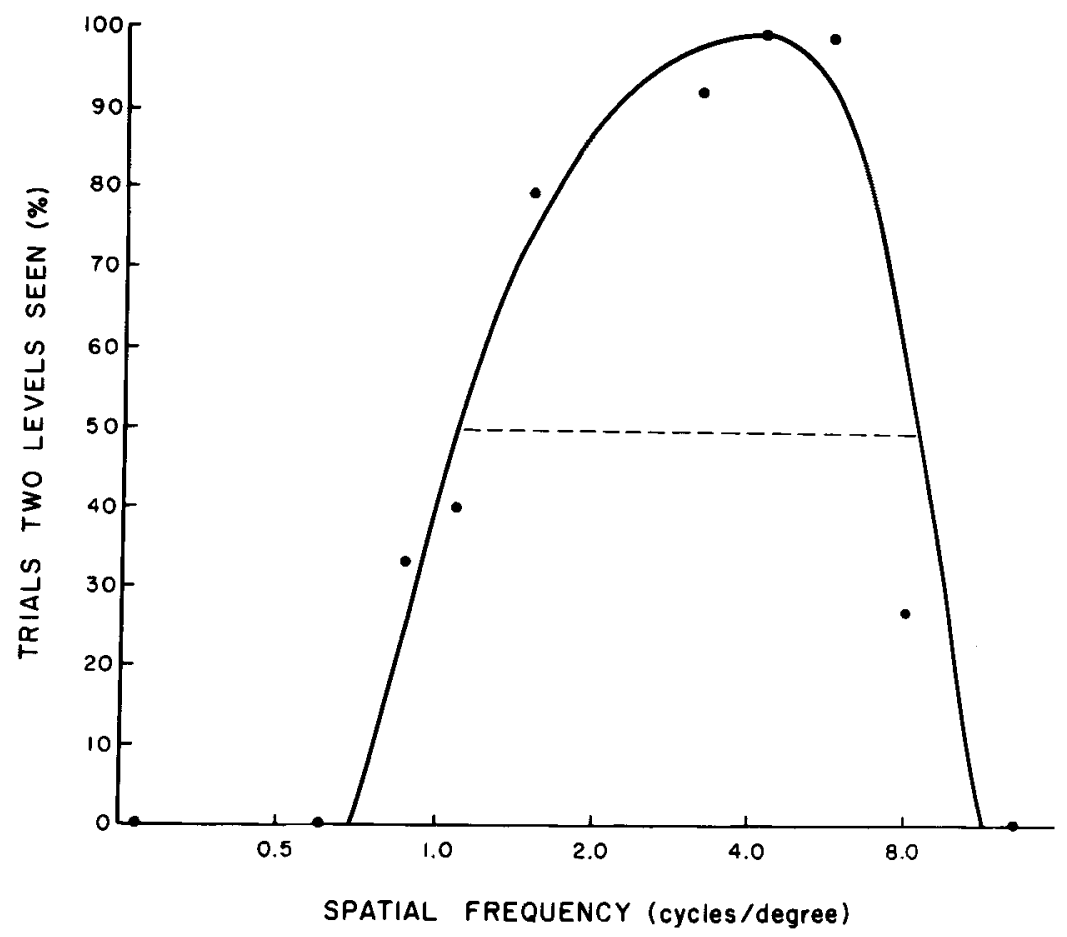

Figure 2. The results of Experiment 1. The probability of each subject's seeing the illusion is presented for all experimental conditions. There were five trials per condition for each subject.

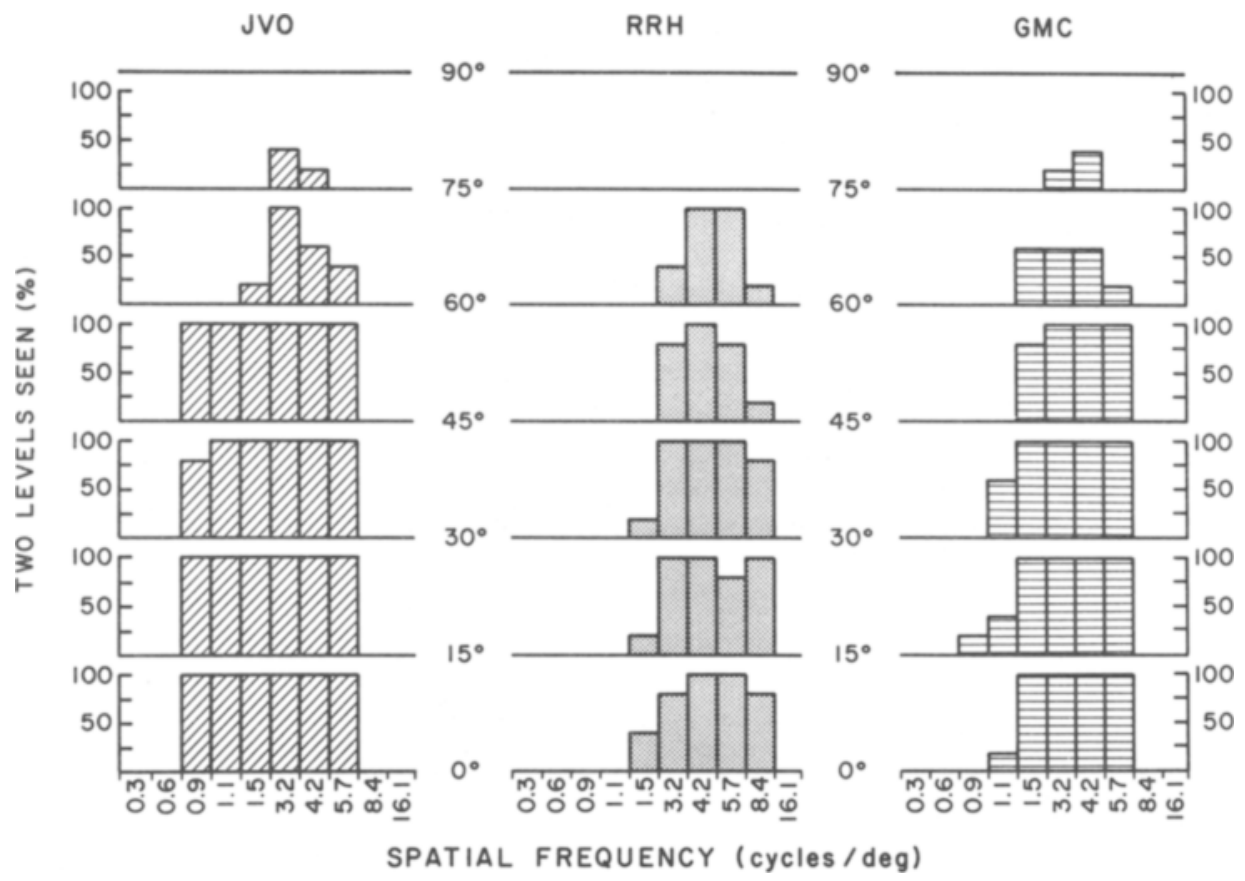

Figure 3. Experiment 1. The probability of seeing the illusion as a function of spatial frequency. The data are for the vertical gratings. Each point is the average of the data for the 3 subjects. 
$3.2 \mathrm{c} / \mathrm{deg}$

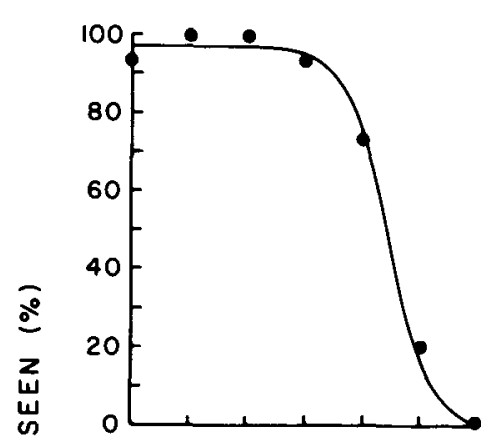

$1.5 \mathrm{c} / \mathrm{deg}$

岕

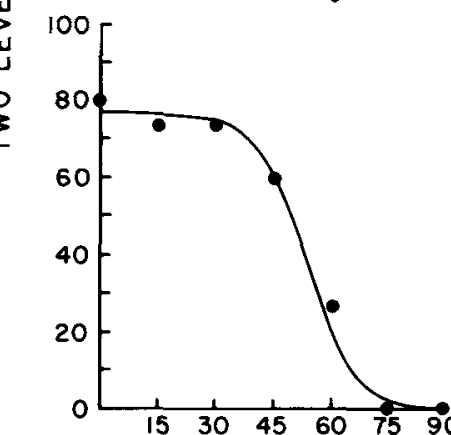

ORIENTATION
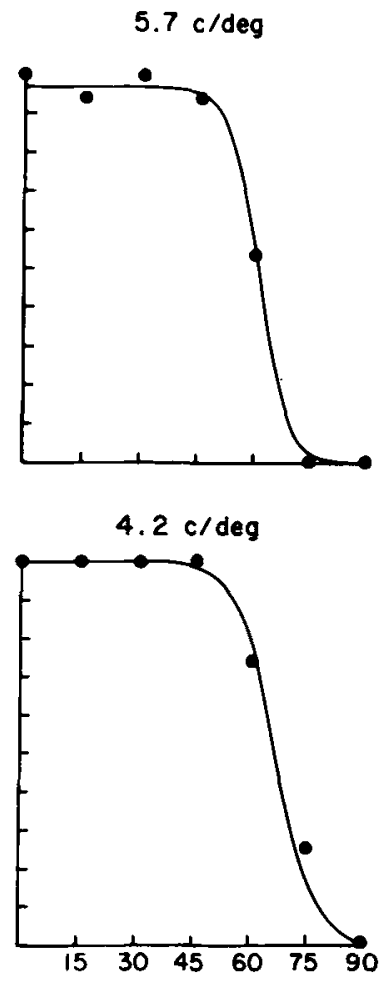

(degrees)

Figure 4. Experiment 1. The probability of seeing the illusion as a function of orientation. The data are for $1.5,3.2,4.2$, and 5.7 cpd in separate panels. Each point is the average of the data for the 3 subjects.

and/or reduced field size might account for our results at these higher spatial frequencies.

\section{EXPERIMENT 2}

\section{Method}

Photographic prints of square-wave gratings, which were presented at a viewing distance of $35.6 \mathrm{~cm}$ (14 in.), had fundamental spatial frequencies of 1.5, 3.2, 4.2, and $5.7 \mathrm{cpd}$. Each grating was presented five times at each of seven field sizes: $5^{\circ}, 10^{\circ}, 14^{\circ}, 20^{\circ}$, $27^{\circ} 48^{\prime}$, and $38^{\circ} 42^{\prime}$. The order of presentation of stimulus conditions was random.

Two of the 3 subjects tested in Experiment 1 served as subjects, J.V.O. and G.M.C. On each of 140 stimulus presentations (4 gratings $\times 7$ field sizes $\times 5$ replications), the subject was asked to indicate the number of levels of depth seen.

\section{Results}

In Figure 6, the means of each subject's data and the grand means are presented. As field size increases, the number of levels seen increases. Multiple levels of depth were not observed if the field size was $10^{\circ}$ or smaller, irrespective of spatial frequency.

The data were analyzed by employing analysis of variance using a randomized block design of 4 (spatial frequency) $\times 6$ (field size) $\times 5$ (replication) in 2 blocks $(2$ subjects). Post hoc tests of significance (Newman-Keuls) were employed to compare means. Spatial frequency $[F(3,3)=14.7, p<.05]$, field size $[F(5,5)=146.6$, $p<.01]$, and the interaction of spatial frequency and field size $[F(15,15)=19.1, p<.01]$ were statistically significant. The number of levels seen increased with each increase in field size $(p>.01)$, except for field sizes of $5^{\circ}$ and $10^{\circ}$, where only one level of depth was seen $(p>.05)$; the number of levels seen was less for the

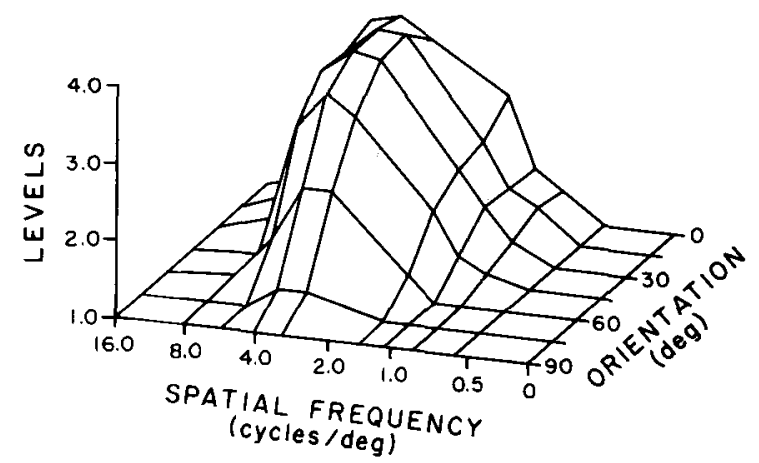

Figure 5. Experiment 1. The number of levels seen as a function of spatial frequency and grating orientation. The mean number of levels seen is presented for each of the experimental conditions. 

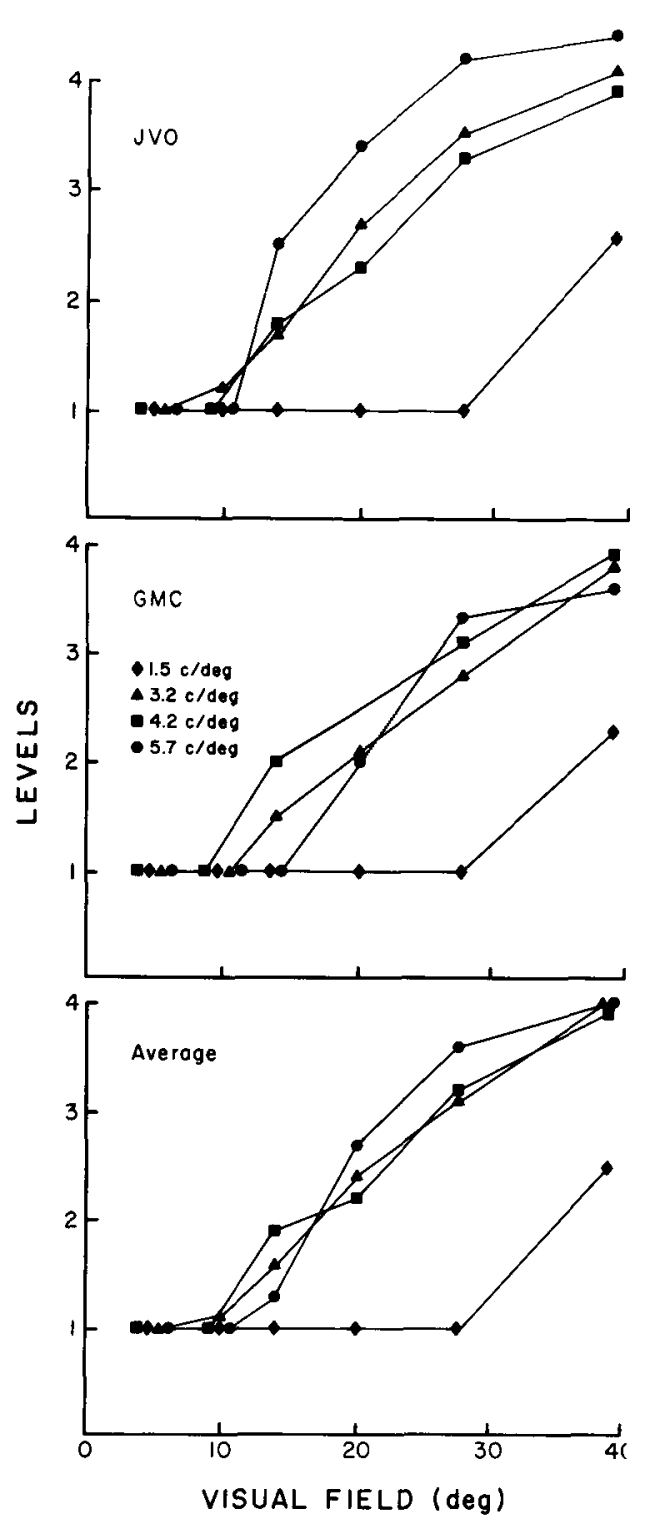

Figure 6. Experiment 2. The number of levels seen as a function of spatial frequency and field size. Points represent means for each subject and the mean of all responses.

1.5-cpd stimuli than for the 3.2-, 4.2-, or 5.7-cpd stimuli at field sizes of $14^{\circ}-32^{\circ} 42^{\prime}$.

Data from Experiment $1\left(43^{\circ}\right.$ field size) for Subjects J.V.O. and G.M.C. were compared with those from Experiment $2\left(38^{\circ} 42^{\prime}\right.$ field size), employing an ANOVA. No effect of field size was observed $[F(1,1)=0.074, p>$ $.10]$, suggesting that the levels of depth increase with increasing field size up to some asymptotic level between $27^{\circ}$ and $43^{\circ}$.

\section{EXPERIMENT 3}

\section{Method}

Viewing distances were varied while spatial frequency and field sizes were kept constant. The spatial frequencies were $3.2,4.2$, and $5.7 \mathrm{cpd}$, and the field sizes were $14^{\circ}, 20^{\circ}$, and $26^{\circ}$. To maintain a spatial frequency of $3.2 \mathrm{cpd}$ with field sizes of $26^{\circ}, 20^{\circ}$, and $14^{\circ}$, distances of $19.6,25.9,35.6$, and $76.7 \mathrm{~cm}$ were employed. To maintain $4.2 \mathrm{cpd}$, distances of $26.9,35.6,48.8$, and $105.2 \mathrm{~cm}$ were employed. To maintain $5.7 \mathrm{cpd}$ at the three field sizes, distances of $35.6,47.0$, and $64.5 \mathrm{~cm}$ were employed. Thirty-five stimulus combinations were presented in random order on five occasions. On each presentation, the subject, J.V.O. or G.M.C., indicated the number of levels of depth seen in the figure.

\section{Results}

In Figure 7, the grand mean and the standard error for the 2 subjects are presented as a function of spatial frequency, field size, and viewing distance. When field size and spatial frequency are held constant, as viewing dis-

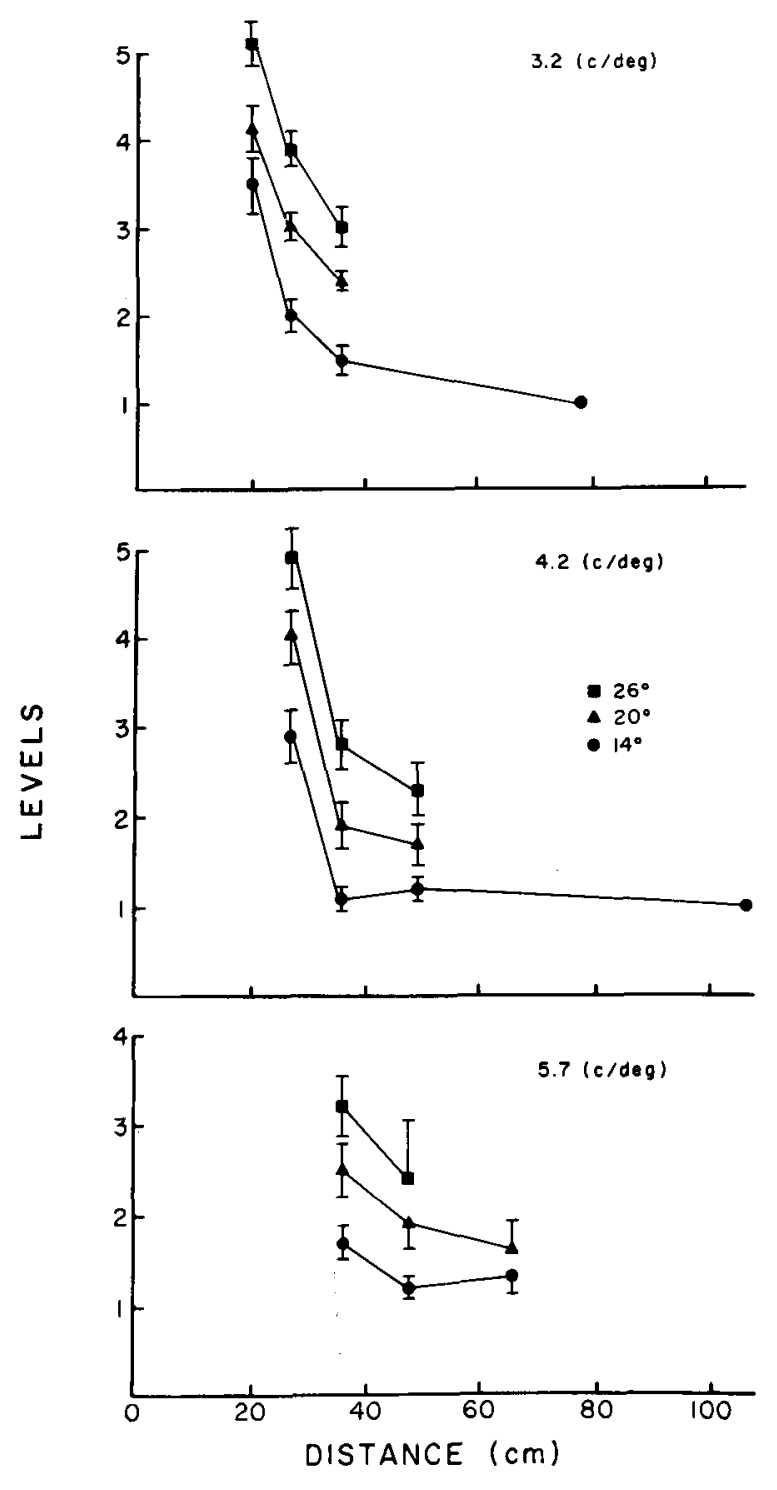

Figure 7. Experiment 3. The number of levels seen as a function of field size, spatial frequency, and viewing distance. Points represent the means across subjects. Bars represent the standard error of all responses. 
tance increases, the number of levels perceived decreases, so only one level of depth is seen at a viewing distance of $70 \mathrm{~cm}$ or greater. For each spatial frequency and distance, as field size is decreased, the number of levels seen is decreased.

As distance increases, the effects of spatial frequency are reduced equally for all field sizes. Thus, at $25.9 \mathrm{~cm}$, there is approximately a 1-level difference between 4.2 and $3.2 \mathrm{cpd}$ at each field size. At $35.6 \mathrm{~cm}$, there is approximately a 0.5 -level difference between 5.7 and $4.2 \mathrm{cpd}$, with $3.2 \mathrm{cpd}$ being intermediate, at each field size. At $47 \mathrm{~cm}$, the difference in the number of levels seen between 5.7 and 4.2 cpd is only 0.1 at each of the three field sizes.

\section{DISCUSSION}

We have presented some fundamental data on the stimulus conditions under which binocularly presented squarewave gratings induce an illusion of depth. The illusion we have described is affected by at least five variables: spatial frequency of the grating, stimulus-field size, viewing distance, stimulus orientation, and viewing condition, either monocular or binocular.

In Experiment 1, we demonstrated that monocularly presented gratings did not induce the illusion. Moreover, the illusion appears to have spatial tuning. As spatial frequency increases, the probability of seeing the illusion, or the number of levels seen, first increases, then decreases. The precise aspects of high-spatial-frequency tuning remain unclear because, to produce the highest spatial frequencies, we had to increase the viewing distance, thereby reducing the field size of the stimulus. Both increased distance and reduced field size were shown in Experiments 2 and 3 to reduce the number of levels seen. Low-spatial-frequency tuning is more certain. Below about $1 \mathrm{cpd}$, the illusion is not seen. The illusion also showed orientation tuning. In general, the probability of seeing the illusion decreased as orientation became more horizontal and, for optimal spatial frequencies, was about $50 \%$ when the grating was oriented about $60^{\circ}$ from the vertical. In Experiment 2, we varied field size and spatial frequency while keeping distance constant. Irrespective of spatial frequency, as field size decreased, the number of levels seen decreased. There was a suggestion that the effects of field size asymptote at about $30^{\circ}$, because, in comparing the results of Experiments 1 and 2, no statistically significant differences were found between the number of levels seen at $43^{\circ}, 38^{\circ}$, and $28^{\circ}$. Therefore, the illusion requires processing in the paracentral region, $7^{\circ}-$ $15^{\circ}$ from fixation. In Experiment 3, spatial frequency, field size, and viewing distance were varied. For each spatial frequency, as field size was decreased or viewing distance increased, the number of levels seen was reduced. For each reduction in field size, the decrement in the number of levels seen was relatively constant, as it was for each increment in distance. The effect of field size diminished as distance increased. The effects of distance asymptoted at about $70 \mathrm{~cm}$, beyond which no illusion was seen irrespective of spatial frequency or field size.

\section{Relationship to Other Depth Illusions: \\ Periodic Stimuli}

Brewster (1844/1983) discovered that if a repetitive stimulus, such as wallpaper or a grating, was examined, the distance from the observer to the plane containing the pattern could be made to appear closer or farther away. Although the difficulty of identifying corresponding portions of the grating presumably causes the wallpaper illusion and the phenomenon we describe, there are a number of differences. Only one level of depth is seen at a time in the wallpaper illusion, and it depends on varying one's angle of convergence. The retinal disparity created by inappropriate convergence may create the illusory depth (Ittelson, 1960; Lie, 1965; Loginenko \& Sokol'skaya, 1975; Ono, Mitson, \& Seabrook, 1971).

Blakemore (1970) observed that if two gratings that differed in spatial frequency were presented dichoptically, one to each eye, the observer had a percept of a single plane tilted in depth. Blakemore's results were reminiscent of the observations by Ogle (1950) of planes tilted in depth when a cylindrical lens was placed in front of one eye or of Wilde's (1950) observations of lines tilted in depth if the dichoptic images consisted of two horizontal stimuli of unequal lengths. Under these conditions, only one tilted plane is observed. Blakemore (1970) also presented a figure suggesting that observation of horizontal sawtooth patterns in depth could be observed. However, he did not explicitly examine the conditions under which this percept occurred. The percept he describes is similar to the one we observe with eccentric fixation of the stimulus.

Boothroyd and Blake (1984) dichoptically presented compound gratings whose two equal-contrast spatialfrequency components carried different disparities. They observed two levels of depth that corresponded, at least qualitatively, to the two disparities present in the compound gratings. Unlike the phenomenon we report, both planes were simultaneously observable throughout the entire visual field.

Piggins (1978) observed that when dichoptically presented gratings of identical spatial frequency but of differing orientation were presented to the two eyes, multiple levels of depth, which resembled a "3-D sawtoothed surface," were observed. He related the effect to moiré fringes. The illusion described by Piggins (1978) is certainly similar to that described by us. Moreover, if one observes vertical gratings binocularly at a relatively close distance, one notes that, seen monocularly, the gratings have a distinct tilt, presumably due to the cyclotorsion of the eyes with convergence. Tyler (1980) observed the same or similar illusion as Piggins (1978). He presented 2-cpd gratings binocularly that reversed at $8 \mathrm{~Hz}$ at a distance of $1 \mathrm{~m}$ with a field size of $10^{\circ}$, and observed a se- 
ries of successive planes. Each plane was $180^{\circ}$ out of phase relative to the plane preceding it. The nearer planes were lower and the more distant planes higher in the visual field. The conditions described by Piggins (1978) and by Tyler (1980) differ substantially from our own. However, the basic characteristics of the perceived phenomena appear similar. Therefore, they probably share common mechanisms.

\section{Probable Mechanisms}

In generating the illusion of multiple levels of depth when gratings are presented binocularly, retinal disparity is an essential element. Establishing relative horizontal disparity as the cue for depth rests on two criteria: the sensation of depth must disappear if (1) one eye is closed and (2) the stimuli are tilted by $90^{\circ}$ so that no horizontal disparities are present. The illusion meets both of these criteria. In Experiment 1, we demonstrated that the sensation of multiple levels of depth disappeared if the gratings were presented monocularly or if the gratings were presented in a horizontal orientation.

Tyler (1980) invoked two mechanisms to account for the illusion observed by Piggins (1978) and himself; the first was fusional displacement (allelotropia), and the second was the tilt of the vertical horopter. Implicit in Tyler's account is ambiguity about position in depth. One prediction derived from Tyler's model is that planes located higher in the visual field should always appear more distant than planes located lower in the visual field. As indicated earlier, when we fixated slightly above eye level, the planes above the locus of fixation appeared closer than the plane at fixation (see Figure 1). These planes appeared to be continuous with planes at the same level below and to the sides of the fixation plane. This observation cannot exclude a role of the vertical horopter, but it indicates that other variables must also operate.

We propose that neural mechanisms responsive to three factors that derive from geometrical optics account for the illusion. These factors are: (1) the problem of correspondence, (2) the horopter (horizontal as well as vertical), and (3) differential projection of the grating stimulus onto the two retinas. Below we shall discuss these factors and their relationship to our results.

It has long been noted that, if different stimuli are presented to the two eyes, there is confusion as to which stimulus elements presented to one eye correspond to the elements presented to the other eye (Julesz, 1971; J. Kepler, 1611, cited in Hirschberg, 1985; Koffka, 1935). The same problem exists if an observer views a stimulus binocularly. Therefore, when a vertical grating is presented binocularly, there is no unique solution to the correspondence problem based on retinal disparity alone. A series of illusory planes exist both in front of and behind the veridical plane (see Appendix, Figure A1). The features of these illusory planes are determined by employing the principles of geometrical optics based on the distance between the nodal points of the two eyes, the viewing distance, and the number of cycles in the pat- tern. The distance of these illusory planes from the veridical plane increases as the spatial frequency of the grating is reduced, as the distance of the observer from the veridical plane increases, or as the distance between the nodal points of the two eyes increases. As the illusory planes become closer to the observer, distances between them decrease. The number of illusory planes increases with the number of grating cycles present in the stimulus. Because the number of cycles is a function of the spatial frequency and field size of the pattern, the number of illusory planes increases as spatial frequency or field size increases. The phase of each successive illusory plane is opposite $\left(180^{\circ}\right.$ out of phase) relative to the ones preceding and following it (see Appendix, Figure A1).

Zero disparity points may be hypothesized to serve as reference points in determining depth (see Shipley \& Hyson, 1972). For example, convergence information may aid in fixing the location of the plane in the region of fixation, since the plane in the region of fixation is always at, or near, the veridical plane. If the plane near fixation is fixed at the veridical plane, then, as a result of the operation of inhibitory mechanisms, one would expect that only the single plane would be seen (Julesz, 1971; Julesz \& Chang, 1976; Marr \& Poggio, 1979; Nelson, 1975), as is observed in Experiment 2 for small field sizes of less than $14^{\circ}$. Therefore, in the foveal region, only one level is seen. However, the fixation point is not the only zero disparity point.

Our use of zero-disparity points as reference points in determining the planes seen is similar to Mitcheson and McKee's (1985) suggestion that specific disparity matching occurs only at disparity discontinuities and that between discontinuities interpolation occurs. Each zerodisparity locus may represent a disparity discontinuity. Evidence of interpolation is less clear in our case.

The horopter is the set of points in visual space that stimulate corresponding points in the retinas; it, therefore, has zero disparity. The horopter has a complicated shape, depending on fixation and other parameters. The horizontal horopter may be approximated by the VeithMueller circle, which is a circle that passes through the nodal point of each eye and the fixation point. The radius of the Veith-Mueller circle, then, is increased as either the distance between the eyes or the distance from the eyes to the fixation point increases. Practically, the viewing distance is the major determinant of the radius of the Veith-Mueller circle. If points of zero disparity can serve as reference points for the correspondence matching process, then each intersection of the horizontal horopter with a false image plane could generate an illusory level. Because the horizontal horopter curves in the periphery from the fixation point toward the observer, it would intersect only those illusory planes that were closer to the observer than the fixation plane.

The quantitative characteristics of the Veith-Mueller circle are easily determined, as are those of the illusory planes (see Appendix). Therefore, the interaction of the two can be determined quantitatively. We computed the 
number of levels an observer could be predicted to see for each of the three experiments. The radius of the horopter decreases with decreased viewing distance. Therefore, if field size and spatial frequency remain fixed, as viewing distance decreases the number of levels intersected would increase, as observed in Experiment 3. As spatial frequency decreases, at some fixed distance the false planes would become more distant from the veridical plane, there would be fewer intersections of the horopter and the false planes, and, therefore, fewer levels of depth would be seen.

A comparison between predicted and obtained results for Experiment 2 and a portion of Experiment 1 is presented in Table A1 of the Appendix. As observed, the predicted number of levels is reduced as spatial frequency and field size are reduced and viewing distance is held constant. Agreement between theoretical predictions and obtained experimental results appears to be very close except for the largest field sizes of $38.7^{\circ}$ and $43^{\circ}$. For the largest field sizes, agreement was poorer as spatial frequency increased. Comparable observations were made when obtained, and predicted results of Experiment 3 were compared.

In the case of larger field sizes, each half of the visual field subtends a larger visual angle on the hemiretina to which it projects in the ipsilateral eye than on the corresponding hemiretina in the contralateral eye. Therefore, each half of the visual field has a different magnification on the hemiretinas. This effect increases as viewing distance decreases or field size increases. In the case of the $43^{\circ}$ field viewed at $35.6 \mathrm{~cm}$, for example, the projection of the right half of the field is $7 \%$ greater in the right than in the left eye and the left half is $7 \%$ greater in the left eye than in the right eye.

Magnification differences have the effect of flattening the horizontal horopter (Ogle, 1950). As the horopter was flattened, it would intersect fewer illusory planes. Consequently, fewer levels of depth would be seen than would be predicted solely on the basis of the characteristics of the Veith-Mueller circle and the correspondence problem. The magnitude of the discrepancy would increase with field size (greater differential magnification) and spatial frequency (more possible intersections with illusory planes for a given field size), as we have observed.

When the field size is large and the viewing distance is short, we have noted that the levels of depth tilt away from the observer. Differential projection of the stimulus on the retinas, with its attendant magnification and spatial frequency differences, should have the effect of generating two symmetrical planes, one to each side of fixation and tilted away from the observer, with their most proximal area being at the area of fixation (Blakemore, 1970; Fiorentini \& Maffei, 1971; Levinson \& Blake, 1979; Ogle, 1950, 1962; Tyler \& Sutter, 1979; Van der Meer, 1978; Wilson, 1976). The slight tilt of the planes may be explained by this phenomenon.

We have noted that the illusion is more clearly seen in the lower hemifield. The superiority of the lower hemifield (upper hemiretina) may be related to the tilt of the vertical horopter (Tyler, 1980) or to the superiority of the upper hemiretinas in processing crossed disparities (Breitmeyer, Julesz, \& Kropfl, 1975).

Several interesting implications derive from our explanation. First, the illusion should be visible with a variety of periodic stimuli. We have noted the illusion with checkerboards and polka dot patterns. Secondly, if the patterns are deformed to coincide with the vertical and horizontal horopter, the illusion should disappear. If the outer edges of the print are curved toward the observer and the top of the print is tilted from the observer, the illusion disappears (Ono, Mitson, \& Seabrook, 1971).

\section{Summary}

We have reported that binocularly presented stationary gratings elicit an illusion of multiple levels of depth that are nonoverlapping and simultaneously visible. These characteristics distinguish the illusion from commonly reported depth illusions. We suggest that the intersection of the horopter with illusory planes generated by the ambiguity of repetitive figures results in the identification of the veridical plane at fixation and subsequent inaccurate solutions in more peripheral areas. This explanation assumes that the nervous system makes use of zero-disparity points as reference points in determining a solution to the correspondence problem, that the zero-disparity reference points are not limited to the central fixation point, and that several reference points may be used simultaneously in different regions of visual space. The effect of magnification differences in the two eyes resulting from large field sizes accounts for deviations of predicted values from obtained experimental data in experimental conditions that employed large field sizes.

The results obtained have several practical and theoretical implications. Many tasks require human observers to watch video screens at relatively close distances. If the monitors are low resolution or if repetitive patterns are contained in the display, the difficulty of subjects' identifying the veridical plane may result in increased fatigue and/or impaired performance. Numerous visual experiments involve observation of suprathreshold repetitive patterns. For example, relatively large visual fields of gratings are employed as stimuli to elicit VEPs. Under conditions in which multiple levels of patterns are observed, the VEPs could be altered.

Many efforts to build an adequate theory of global stereopsis have concentrated on finding a model or algorithm that correctly solves the correspondence problem (Julesz, 1971; Julesz \& Chang, 1976; Marr \& Poggio, 1979; Mayhew, 1983; Nelson, 1975). However, if an algorithm is to describe human stereopsis adequately, it must predict the inaccurate as well as the accurate solutions of the visual system to the correspondence problem. Experiments 1, 2, and 3 provide quantitative information about a set of conditions under which incorrect solutions to the correspondence problem are found. Our explanation of the illusion emphasizes the importance of zero- 
disparity reference points, whether in the area of fixation or the periphery, in aiding the nervous system to solve the correspondence problem.

\section{REFERENCES}

Berkson, J. (1951). Why I prefer logits to probits. Biometrics, 38, 327-339.

Blakemore, C. (1970). A new kind of stereoscopic vision. Vision Research, 10, 1181-1199.

BOOTHROYD, K., \& BLAKE, R. (1984). Stereopsis from disparity of complex grating patterns. Vision Research, 24, 1205-1222.

BretrmeYer, B., Julesz, B., Kropfl, W. (1975). Dynamic randomdot stereograms reveal up-down anisotropy and left-right isotropy between cortical hemifields. Science, 187, 269-270.

BREWSTER, D. (1983). On the knowledge of distance given by binocular vision. In N. J. Wade (Ed.), Brewster and Wheatstone on vision. London: Academic Press. (Reprinted from Transactions of the Royal Society of Edinburgh, 1844, 15, 663-674)

Fiorentini, A., \& MAFFeI, L. (1971). Binocular depth perception without geometrical cues. Vision Research, 11, 1299-1305.

ITTELSON, W. H. (1960). Visual space perception. New York: SpringerVerlag.

Julesz, B. (1971). Foundations of cyclopean perception. Chicago: University of Chicago Press.

JuLesz, B., \& CHANG, J. J. (1976). Interaction between pools of binocular disparity detectors tuned to different disparities. Biological Cybernetics, 22, 107-120.

KoffKa, K. (1935). Principles of gestalt psychology. New York: Harcourt Brace.

LeVINSON, E., \& BLAKE, R. (1979). Stereopsis by harmonic analysis. Vision Research, 19, 73-78.

LIE, I. (1965). Convergence as a cue to perceived size and distance. Scandinavian Journal of Psychology, 6, 109-116.

LoginenKo, A., \&OKOL'SKaYA, T. (1975). Leont'ev's phenomenon: The effect of distance and spacing. Soviet Psychology, 14, 75-96.

MARR, D., \& PoGgio, T. (1979). A computational theory of human stereo vision. Proceedings of the Royal Society of London B, 204, 301-328.

MAYHEW, J. (1983). Stereopsis. In O. J. Braddick \& A. C. Sleigh (Eds.), Physiological and biological processing of images. Berlin: SpringerVerlag.

MrTCheson, G. J., \& McKeE, S. P. (1985). Interpolation in stereoscopic matching. Nature, 315, 402-404.

NeLSON, J. I. (1975). Globality and stereoscopic fusion in binocular vision. Journal of Theoretical Biology, 49, 1-88.

OGIE, K. N. (1950). Researches in binocular vision. New York: Hafner.

OGLE, K. N. (1962). Special topics in binocular spatial localization. In H. Davson (Ed.), The eye: Vol. 4. Visual optics and the optical space sense. New York: Academic Press.

Ono, H., Mrtson, L., \& SeabrooK, K. (1971). Change in convergence and retinal disparities as an explanation for the wallpaper phenomenon. Journal of Experimental Psychology, 91, 1-10.

Piggins, D. (1978). Moirés maintained internally by binocular vision. Perception, 7, 679-681.

REED, L. J., BERKSON, J. (1929). The application of the logistic function to experimental data. Journal of Physical Chemistry, 33, 760-779.

SHIPLEY, T., \& Hyson, M. (1972). The stereoscopic sense of order-a classification of stereograms. American Journal of Optometry and Ar chives of the American Academy of Optometry, 49, 83-96.

TYLER, C. W. (1980). Binocular moire fringes and the vertical horopter. Perception, 9, 475-478.

Tyler, C. W., \& Sutrer, E. (1979). Depth from spatial frequency difference: An old kind of stereopsis? Vision Research, 19, 859-865.

VAN DER MEER, H. C. (1978). Linear combinations of stereoscopic depth effects in dichoptic perception of gratings. Vision Research, 18, 707-714.

WILDE, K. (1950). Der Punktreiheneffekt und die Rolle der binokularen Querdisparation beim Tiefensehen. Psychologische Forschung, 23, 223-262.
WIISON, H. R. (1976). The significance of frequency gradients in binocular grating perception. Vision Research, 16, 983-989.

\section{APPENDIX}

It is well known that when disparate images are presented to the two eyes, it is not obvious which of the elements of one image correspond to the elements of the other (Julesz, 1971; Koffka, 1935). It is less frequently recognized that the same difficulty exists if the same pattern is presented to both eyes. When a vertical square-wave grating is presented to a subject, there are a number of these false images in front of and behind the real plane (Figure A1). The location and number of these false images can be drawn using geometrical principles. If the real plane includes a grating of $n$ cycles, then the number of images equals $2(n-1)$, where $n-1$ images are in front of the real plane and $n-1$ are behind it. As Figure A1 shows, they may be labeled $\pm 1, \pm 2, \pm 3, \ldots, \pm n-1$, where the positive numbered images are in front of the real plane and the negative numbered images are behind the real plane. The first image is closest to the real plane. The others are farther away from it. It can be seen that the $i$ th image includes $n-i$ grating cycles and that the gratings in two adjacent images are in counterphase.

The distance between each image and the subject can be determined as follows.

We shall assume that fixation is in the center of the plane at eye level. Therefore, a symmetrical perception occurs and the discussion may be conveniently limited to one half of the figure.

In Figure A2, where $c=$ the physical size of one cycle square wave $(\mathrm{cm} / \mathrm{c}), \mathrm{T}=$ width of plane $(\mathrm{cm}), \mathrm{m}$ and $\mathrm{n}=$ nodal points of right eye and left eye, $\mathrm{O}=$ fixation point (center of plane), $\mathrm{h}=$ distance between $\mathrm{m}$ and $\mathrm{n}(\mathrm{cm}), \mathrm{d}=$ viewing distance $(\mathrm{cm})$, and $\mathrm{li}=$ distance between $i$ th image and subject, the first image intersected $O I$ at $P_{1}, P_{1} I=L_{1}, I n, O P_{1} M_{1}$, and $L_{n} I P$.

$$
\frac{\mathrm{OP}_{1}}{\mathrm{OM}_{1}}=\frac{\mathrm{IP}}{\mathrm{IL}_{\mathrm{n}}} \quad \text { i.e., } \frac{\mathrm{d}-\mathrm{I}_{1}}{\mathrm{C} / 2}=\frac{1}{\mathrm{~h} / 2} \text {. }
$$

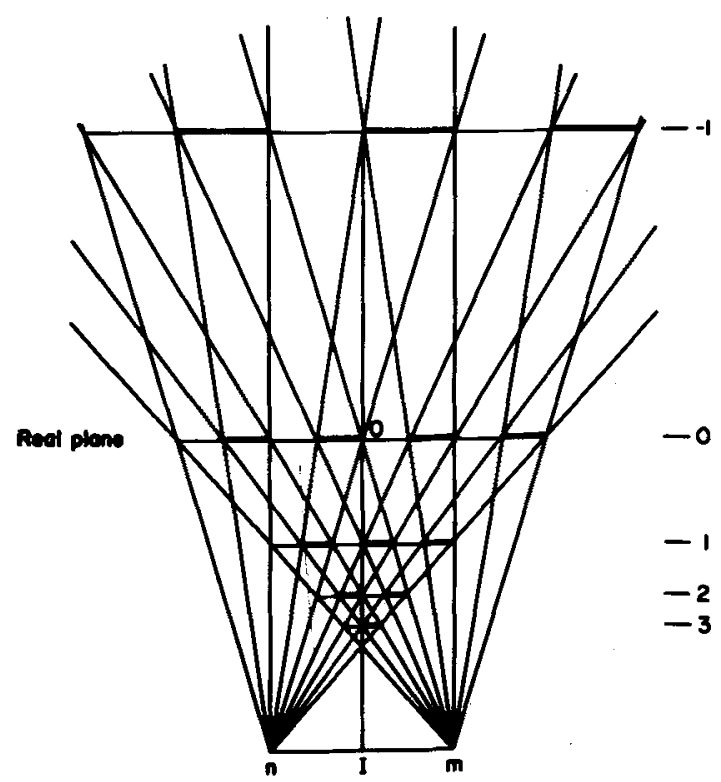

Figure A1. Correspondence problem. A repetitive pattern presented binocularly generates false images in front of and behind the real pattern. Only one of the four images behind the pattern has been drawn. 
So $l_{1}=d[h /(h+c)]$. The second image intersected OI at $P_{2}$; therefore $P_{2} I=l_{2}$. Considering $O P_{2} M$ and $\mathrm{INP}_{2}$, we can get $l_{2}=$ $\mathrm{d}[\mathrm{h} /(\mathrm{h}+2 \mathrm{c})]$.

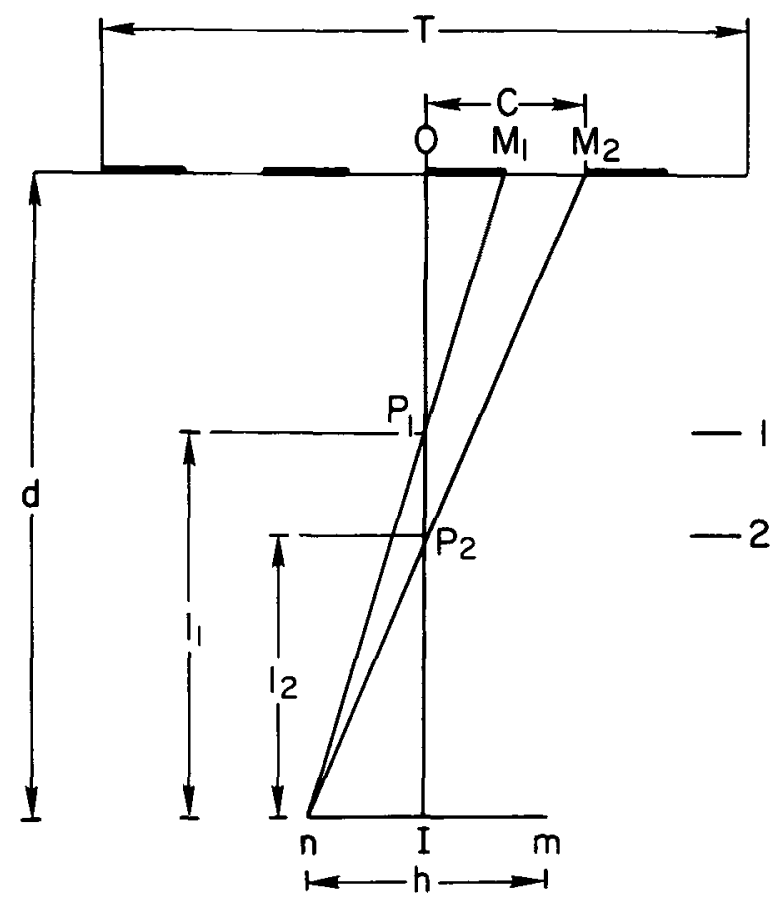

Figure A2. Lacation of false images. The figure presents the geometrical considerations that determine the location of the false images.

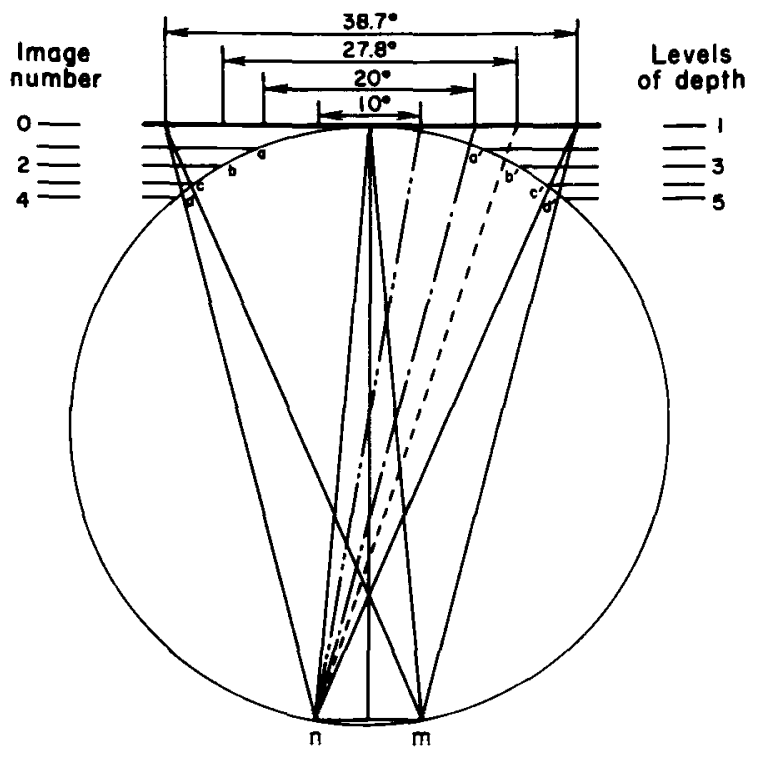

Figure A3. Intersection of Veith-Mueller circle with false images. The Veith-Mueller intersects with the false images that result from the ambiguity of correspondence when repetitive patterns are presented binocularly. The figure is drawn to scale. Reducing field size reduces the number of planes intersected.
Table A1

Comparison of Predicted and Mean Experimental Results

\begin{tabular}{|c|c|c|c|c|c|c|c|c|}
\hline \multirow[b]{3}{*}{ Field Size } & \multicolumn{8}{|c|}{ Spatial Frequency } \\
\hline & \multicolumn{2}{|c|}{$1.5 \mathrm{cpd}$} & \multicolumn{2}{|c|}{$3.2 \mathrm{cpd}$} & \multicolumn{2}{|c|}{$4.2 \mathrm{cpd}$} & \multicolumn{2}{|c|}{$5.7 \mathrm{cpd}$} \\
\hline & Exp. & Pred. & Exp. & Pred. & Exp. & Pred. & Exp. & Pred \\
\hline $5^{\circ}$ & 1 & 1 & 1 & 1 & 1 & 1 & 1 & 1 \\
\hline $10^{\circ}$ & 1 & 1 & 1 & 1 & 1 & 1 & 1 & 1 \\
\hline $14^{\circ}$ & 1 & 1 & 1.5 & 1 & 2 & 1 & 1 & 1 \\
\hline $20^{\circ}$ & 1 & 1 & 2.1 & 1 & 2 & 2 & 2.2 & 2 \\
\hline $27.8^{\circ}$ & 1 & 1 & 2.8 & 2 & 3.1 & 3 & 3.4 & 3 \\
\hline $38.7^{\circ}$ & 2.3 & 2 & 3.8 & 4 & 3.9 & 5 & 3.6 & 6 \\
\hline $43^{\circ}$ & 2.5 & 2 & 3.2 & 5 & 3.4 & 6 & 3.3 & 7 \\
\hline
\end{tabular}

Note-Predicted results were calculated using the definition of the VeithMueller circle and Equation A1. An interpupillary distance of $64 \mathrm{~mm}$ was assumed. Experimental results were the mean of the 2 observers in Experiment 2. Exp. = experimental values obtained; Pred. = predicted values.

In terms of the same argument, the $n$th image positioned in $P_{n}$ and $P_{n} I=l_{n}$ :

$$
l_{n}=d \frac{h}{h+n c},
$$

where $n=0, \pm 1, \pm 2, \pm 3, \pm T / c$. When $n=0,1_{0}=d$, it is the position of real plane; $n>1$ images are in front of real plane, and $n<1$ images are behind real plane.

The visual angle (A) of the section between $(i-1)$ th and $i$ th images can be obtained by the formula:

$$
A=2 \tan ^{-1} \frac{h+i c}{2 d}-2 \tan ^{-1} \frac{h+c(i-1)}{2 d}
$$

\section{Horopter}

The loci of points in space which stimulate exactly corresponding points on the two retinas is the horopter. If one makes certain simplifying assumptions, the horizontal horopter is a circle that passes through the nodal point of each eye and the fixation point. This theoretical horizontal horopter is known as the VeithMueller circle. As the distance between the nodal points may be considered constant, the major determinant of the radius of the Veith-Mueller circle is the distance of the subject's eyes from the fixation point. If the observed figure is a repeating pattern, such as a vertical grating, the horopter will intersect the false images generated by the ambiguity of correspondence in the figure. Figure A3 illustrates the intersection of the Veith-Mueller circle with the false images. It is drawn to scale, assuming a $35-\mathrm{cm}$ viewing distance and a fundamental spatial frequency of $3.2 \mathrm{cpd}$. The positions of the false images were calculated using Formula A1. Four field sizes were considered. Using these principles, one can determine the number of false images that intersect with the Veith-Mueller circle. We have assumed that the number of intersected planes predicts the number of levels of depth that a subject would see. In Table A1, we present the mean experimental values obtained in Experiment 2 and compare them with the predicted results obtained using the principles illustrated in Figure A3. The agreement is generally quite close, except for the largest field size, where the magnitude of the difference increases as spatial frequency increases.

Manuscript received September 12, 1986; revision accepted for publication March 2, 1987.) 